\title{
Metagenomic Analysis of Intestinal Microflora in Rats Treated with Ellagic Acid and Sinapic Acid
}

Hamdullah ( $\square$ hamdullahseckin@yyu.edu.tr)

\section{Research Article}

Keywords: Ellagic Acid, Sinapic Acid, Gut microbiota, Metagenome, 16 S rDNA

Posted Date: November 1st, 2021

DOI: https://doi.org/10.21203/rs.3.rs-1034135/v1

License: (9) (i) This work is licensed under a Creative Commons Attribution 4.0 International License. Read Full License 


\section{Abstract}

The correct functioning of the intestinal mechanism is very important for human health. In particular, the microecosystem of the gut has a vital role in the development of the host and the continuity of metabolism. For this reason, many studies have been conducted on the intestinal microbiota. Today, the use of phenolic compounds in the production of complementary and alternative medicines is quite common. In our study, changes in intestinal microflora at the species level were evaluated in Wistar Albino rats treated with Ellagic Acid and Sinapic Acid. The first group was formed as the control group. Ellagic Acid (EA) was given to the second group of rats $(50 \mathrm{mg} / \mathrm{kg} / \mathrm{d})$. The third group of rats was given Sinapic Acid (SA) $(20 \mathrm{mg} / \mathrm{kg} / \mathrm{d})$. Fourth group of rats were given Ellagic Acid EA ( $50 \mathrm{mg} / \mathrm{kg} / \mathrm{d})$ and Sinapic Acid SA (20 mg/kg/d). At the end of the study, intestinal tissues were taken under appropriate sterile conditions. The V3-V4 points located in the $16 \mathrm{~S}$ rDNA gene regions of the bacteria in the microflora were replicated by the specific primers developed in accordance with these regions. The data obtained as a result of sequencing analyzes were evaluated comparatively for four groups. As a result, it was determined that Ellagic Acid and Sinapic Acid have a positive effect on probiotic microorganisms (Lactobacillus) in the intestine. It was also determined that while it caused a decrease in the rate of some pathogenic microorganisms such as Streptococcus, it caused an increase in the number of some pathogens.

\section{Introduction}

Intestine; It is a metabolic organ that has very important roles such as digestion, absorption of nutrients, energy need, regulation of natural and adaptive immune functions, vitamin synthesis and resistance to diseases and hosts active microorganisms (Sekirov et al., 2010; Rooks and Garrett, 2016; Lin et al., 2018). Symbiotic bacteria that lives in the our intestine are important for the intestinal system. There is a continuous metabolic mechanism of interaction between the host and the microbiota (Levy et al., 2017). It is well known that the colonization of microorganisms in the host gut is affected by factors related to host and non-host interactions (Yan et al., 2016). The composition of the gut microbiota is affected by factors such as diet, antibiotic therapy, maternal microbiota, and genotype (Lozupone et al., 2012). In the human gastrointestinal tract, the vast majority of the microbiota plays a critical role in maintaining health throughout life (Gill et al., 2006). Intestinal flora varies greatly between individuals. And it is thought to play a key role in the diagnosis of many diseases (Ayres, 2016). Dysfunction of the microbiome-brain-gut axis plays a role in stress-related disorders such as irritable bowel syndrome (IBS), depression and anxiety, and neurodevelopmental disorders such as autism (Borre et al., 2014).

Many phytochemicals are used in the protection of human health. Among them, the use of phenolic compounds is quite common. In recent years, the use of phenolic structures such as Ellagic Acid and Sinapic Acid as material in scientific studies has increased. Ellagic acid (EA), also known as trihydroxy acid ( $\mathrm{C} 14 \mathrm{H} 6 \mathrm{O} 8)$, is generally found in red and purple fruits such as raspberry, strawberry, pomegranate, grape, and blackcurrant (Grundhofer et al., 2001; Zhou et al., 2019). It has focused on the contribution of EA consumption to healthy life, especially on studies investigating cancer prevention and its anti- 
inflammatory and antidiabetic effects (Rios et al., 2018; Ceci et al., 2018; Polce et al., 2018). Another phenolic compound, Sinapic Acid (3,5-dimethoxy-4-hydroxycinnamic acid), is a derivative of hydroxycinnamic acid. Found in fruits, vegetables, grains, plant seeds and some spices, SA has antioxidant, antimicrobial, anti-inflammatory, anticancer, chemopreventive and antiapoptotic activity. (Chen, 2016; Hameed et al., 2016; Bin Jardan et al., 2020).

Molecular diagnostic tests and next-generation metagenomic approaches analyze all the Nucleic Acids present in a sample, enabling the analysis of the microbiome with a wide range of pathogens such as parasites, bacteria, viruses (Lefterova et al., 2015; Udayangani et al., 2017). Studying the gut microbiota has become easier due to rapid advances in microarray technology and statistical analysis of data. For this reason, it is known that genomic analyzes make important contributions to phylogenetic studies. With the emergence of next-generation sequencing technologies, sequence analysis of the 16S rDNA gene region has become a culture-independent approach to diagnose bacterial communities (Morgan and Huttenhower, 2014; Tarnecki et al., 2017).

In our study, it was aimed to reveal positive or negative results that may occur in bacterial species in the intestinal composition in rats treated with Ellagic Acid and Sinapic Acid. The first group was formed as the control group. The second is the group administered Ellagic Acid. The third group was determined as the group that was administered Sinapic Acid and the fourth group was given Ellagic Acid and Sinapic Acid. Sequence analyzes of the V3-V4 points of the $16 \mathrm{~S}$ rDNA gene regions were performed to evaluate the metagenomic data in a comparative way.

\section{Material And Method}

\section{Diets and Rats}

28 female Wistar Albino rats used in this study were obtained from Van Yüzüncü Yıl University Experimental Medicine Application and Research Center. Rats were housed in rooms under normal light and dark cycles $\left(21 \pm 2^{\circ} \mathrm{C}\right)$. The feed and water of the rats kept in suitable cages were given in accordance with the standards (Ad libitum). All stages of the study were approved by Van Yüzüncü Yıl University Animal Experiments Local Ethics Committee (Approval number: 28 March 2019/03).

A total of 28 rats were used in this study. Rats were randomly divided into four groups $(n=7)$.

1. Control (C) group: They were fed with standard rat food and water until the end of the experiment. No further action was taken.

2. Ellagic Acid (EA) group: Rats were given $E A(50 \mathrm{mg} / \mathrm{kg} / \mathrm{d})$.

3. Sinapic Acid (SA) group: Rats were given $S A(20 \mathrm{mg} / \mathrm{kg} / \mathrm{d})$.

4. Ellagic Acid + Sinapic Acid (SA + EA) group: Rats were given SA $(20 \mathrm{mg} / \mathrm{kg} / \mathrm{d})$ and EA $(50 \mathrm{mg} / \mathrm{kg} / \mathrm{d})$ (Raish et al., 2018; Farbood et al., 2019; Altındağ et al., 2021).

At the end of the study period, the rats, which were deprived of feed for 12 hours, were rendered dead by direct cannulation of the heart after the administration of $50 \mathrm{mg} / \mathrm{kg}$ ketamine. Intestinal tissues of the 
rats were taken and stored at $-20^{\circ} \mathrm{C}$ until the study.

\section{Isolation of Bacteria and Genetic Analysis}

Isolation of bacteria in the intestinal flora, V3, V4 region analyzes of the 16S rDNA gene were performed by BM Labosis using the QIIME2 procedure. DNA isolation and quality control were performed from the samples to create a library.

The V3-V4 region of the $16 \mathrm{~S}$ rDNA gene from the isolated target DNAs was amplified using specific primers $\mathrm{F}$ (5'-TCGTCGGCAGCGTCAGATGTGTATAAGAGACAGCCTAC GGGNGGCWGCAG-3') and R (5'GTCTCGTGGGCTCGGAGATGTGTATAAGAGAG AGGACTACHV GGGTATCTAATCC-3'). Then purification was done. During the PCR step, indexes and adapters were added using the index kit (Nextera XT). After purification, the concentration of the libraries created by Real-time PCR was measured. It was then diluted to $4 \mathrm{nM}$ and normalized. Normalized samples were brought together by the pooling method. After sieving particles, primers and barcodes smaller than twenty nucleotides; Species identification was performed for taxonomic analyses.

\section{Results}

\section{Species Level Comparative Analysis Results}

Taxonomic analysis of bacteria in the intestinal microflora of all groups was performed. The data generated as a result of the metagenomic analysis of the $\mathrm{V} 3, \mathrm{~V} 4$ region of the $16 \mathrm{~S}$ rDNA gene point were examined comparatively. The taxonomic column chart created for the species level is given in Figure 1. The comparative analysis of bacteria and their ratios obtained as a result of metagenome analysis of all groups are given in Table 1. 
Table 1

Taxonomy Table for Species Level

\begin{tabular}{|c|c|c|c|c|}
\hline Bacteria species & $\begin{array}{l}\text { Control group } \\
(\%)\end{array}$ & $\begin{array}{l}\text { EA group } \\
(\%)\end{array}$ & $\begin{array}{l}\text { SA group } \\
(\%)\end{array}$ & $\begin{array}{l}\text { SA+EA group } \\
(\%)\end{array}$ \\
\hline Actinomyces bovis & 7,658 & 0,157 & 0,111 & 0,104 \\
\hline Bifidobacterium adolescentis & 0,229 & 0,714 & 0,019 & 0,083 \\
\hline Corynebacterium ulcerans & 0,244 & 0,036 & 0 & 0 \\
\hline Rothia terrae & 2,087 & 0 & 0 & 0,069 \\
\hline Parvibacter caecicola & 0,070 & 0,235 & 0 & 0,586 \\
\hline Bacteroides dorei & 0,298 & 2,084 & 0,184 & 0,223 \\
\hline Bacteroides vulgatus & 0,394 & 0,790 & 0,044 & 0 \\
\hline Prevotella copri & 1,759 & 6,256 & 0,206 & 0,565 \\
\hline Parabacteroides distasonis & 0,036 & 0,243 & 0 & 0 \\
\hline Parabacteroides merdae & 0,088 & 0,481 & 0,025 & 0,146 \\
\hline Bacillus vallismortis & 1,313 & 0,911 & 0 & 0,041 \\
\hline Gemella parahaemolysans & 3,703 & 0,364 & 0,098 & 0,076 \\
\hline Staphylococcus succinus & 0 & 0,251 & 0 & 0 \\
\hline Vagococcus fessus & 0,331 & 0,031 & 0 & 0 \\
\hline Vagococcus lutrae & 0,560 & 0,120 & 0 & 0,048 \\
\hline Lactobacillus delbrueckii & 2,652 & 14,655 & 1,063 & 2,449 \\
\hline Lactobacillus faecis & 0,291 & 0,364 & 0,111 & 0,362 \\
\hline Lactobacillus intestinalis & 0,225 & 0,678 & 0,050 & 1,611 \\
\hline Lactobacillus johnsonii & 0,442 & 3,053 & 0,387 & 8,651 \\
\hline Lactobacillus murinus & 0,265 & 0,141 & 0,082 & 0,495 \\
\hline Lactobacillus reuteri & 0,106 & 0,481 & 0 & 0,327 \\
\hline Lactobacillus satsumensis & 0,202 & 0,625 & 0,038 & 0 \\
\hline Streptococcus danieliae & 25,729 & 8,053 & 0 & 0,341 \\
\hline Streptococcus hyointestinalis & 0,811 & 0 & 0 & 0,118 \\
\hline Streptococcus sanguinis & 29,005 & 1,005 & 0,279 & 0,334 \\
\hline
\end{tabular}

Other species (The total of species below $0.2 \%$ in four groups) 


\begin{tabular}{|c|c|c|c|c|}
\hline Bacteria species & $\begin{array}{l}\text { Control group } \\
(\%)\end{array}$ & $\begin{array}{l}\text { EA group } \\
(\%)\end{array}$ & $\begin{array}{l}\text { SA group } \\
(\%)\end{array}$ & $\begin{array}{l}\text { SA+EA group } \\
\text { (\%) }\end{array}$ \\
\hline Streptococcus thermophilus & 0,317 & 0,948 & 0,215 & 0,244 \\
\hline Clostridium disporicum & 0,162 & 0,225 & 0 & 0 \\
\hline Clostridium sporogenes & 0,280 & 0,054 & 0 & 0 \\
\hline Eubacterium coprostanoligenes & 0,147 & 0,219 & 0 & 1,179 \\
\hline Blautia luti & 0,121 & 3,077 & 0,060 & 0,216 \\
\hline Kineothrix alysoides & 0,066 & 0,817 & 0,047 & 6,398 \\
\hline Romboutsia timonensis & 6,647 & 3,949 & 0,133 & 2,721 \\
\hline Ethanoligenens harbinense & 0,284 & 0,893 & 0,028 & 0 \\
\hline Faecalibacterium prausnitzii & 0,608 & 2,142 & 0,079 & 0 \\
\hline $\begin{array}{l}\text { Pseudoflavonifractor } \\
\text { phocaeensis }\end{array}$ & 0,025 & 0 & 0 & 5,253 \\
\hline Faecalibaculum rodentium & 0,118 & 0,261 & 0 & 0,348 \\
\hline Veillonella atypica & 1,184 & 0,052 & 0,015 & 0 \\
\hline Veillonella rogosae & 1,161 & 0,049 & 0 & 0 \\
\hline Mesorhizobium shangrilense & 0,036 & 0,217 & 0 & 0 \\
\hline Acetobacter indonesiensis & 0,081 & 0,411 & 0 & 0 \\
\hline Gluconacetobacter liquefaciens & 0,309 & 1,031 & 0,098 & 0,055 \\
\hline Zymomonas mobilis & 0,188 & 0,720 & 0,034 & 0,083 \\
\hline Campylobacter jejuni & 0,844 & 5,965 & 0 & 0 \\
\hline Helicobacter cholecystus & 0,044 & 2,613 & 0 & 0 \\
\hline Helicobacter ganmani & 1,730 & 17,620 & 24,031 & 22,125 \\
\hline Helicobacter pylori & 0 & 0,246 & 0,031 & 0 \\
\hline Helicobacter rodentium & 0 & 0,075 & 46,342 & 25,140 \\
\hline Escherichia coli & 0,284 & 0,403 & 0,025 & 0 \\
\hline Mycoplasma muris & 0 & 0,026 & 23,828 & 0,907 \\
\hline Other species & 6,866 & 16,259 & 2,337 & 18,751 \\
\hline
\end{tabular}




\section{Discussion}

The intestinal area, which contains many species, accounts for about $2 \mathrm{~kg}$ of body weight. It is estimated that the amount of microorganisms it contains is 10 times higher than the number of cells in the human body (Peng and Biswas, 2017). Comprehensive analysis of the genetic material of microorganisms results rapidly using next-generation sequencing (mNGS), and metagenomic research. Metagenomic analyzes; It facilitates the diagnosis and treatment of many infectious diseases because it has a wide scope such as gene expression, antimicrobial resistance, microbiome, and oncology (Chiu and Miller, 2019). Metagenomic studies contribute to the diagnosis of beneficial and harmful bacteria in the body with the help of $16 \mathrm{~S}$ rDNA gene sequencing technology. In our study, we determined the change in the diversity of microorganisms due to the use of Ellagic Acid and Sinapic Acid in the intestinal microbiota as a result of metagenome analysis.

Metagenome analysis of body microbiota is usually done with stool samples or small intestinal fluid. (Deatherage and Cookson, 2012). In our study, rat intestine was used for metagenome analysis. Bifidobacterium, Lactobacillus, Bacteroides spp. Compounds produced by commensal bacteria, such as commensal bacteria, inhibit the growth of some intestinal pathogens (Sorbara and Pamer, 2019). It is observed that Lactobacillus species (Lactobacillus delbrueckii, Lactobacillus faecis, Lactobacillus intestinalis, Lactobacillus johnsonii, Lactobacillus reuteri, Lactobacillus satsumensis), which is one of the most important bacterial groups in the intestinal ecosystem, showed a significant increase in the group using Ellagic acid compared to the control group. On the other hand, there was a decrease in the ratio of Lactobacillus species in the group in which Sinapic acid was used. The rate of Vagococcus (Vagococcus fessus and Vagococcus lutrae) species that cause disease in humans in some cases decreased in the groups using Sinapic acid and Ellagic acid compared to the control group. (Lawson et al. 1999). In rats with ethanol-induced ulcers, pretreatment with Sinapic Acid was found to decrease the acidity rate and reduce the stiffness of injuries in the gastric mucosa (Raish et al., 2021). Ellagic Acid taken into the body through food or dietary supplements has been shown to have the potential to be used in reducing intestinal inflammation (Iglesiasa et al., 2020).

In rats treated with Gallic and Ellagic acid, diltiazem permeability was found to be significantly increased in the ileum (Athukuri and Neerati, 2017). The stomach is the main place where Helicobacter species are located. However, they can cause infection in any part of the gastro-intestinal system where gastric epithelial cells are present (Makola et al., 2007). In our study, it was observed that Helicobacter species (Helicobacter cholecystus, Helicobacter ganmani, Helicobacter pylori, Helicobacter rodentium) increased in groups using Ellagic Acid and Sinapic Acid.

Streptococci, Enterococci, Mycoplasma and Staphylococci species in Firmicutes phylum are generally pathogenic (Becattini et al., 2016). Streptococcus species (Streptococcus danieliae, Streptococcus hyointestinalis, Streptococcus sanguinis), which had a significant proportion in the control group, were significantly reduced in the groups using Ellagic Acid and Sinapic Acid. Mycoplasma muris, found in the mouse genital tract, is a pathogenic bacterium (Zinatizadeh et al., 2018). It was observed that the rate of 
Mycoplasma muris $(23,828 \%)$ increased significantly in rats treated with sinapic acid. The amount of Escherichia coli bacteria, which is the source of infection in the intestinal system, increased approximately two times in the group using ellagic acid compared to the control group. Campylobacter jejuni (Fujimoto and Amako, 1990) bacteria, one of the most common causes of gastroenteritis, showed a high increase (5.965\%) in rats treated with Ellagic Acid. Veillonella strains are symbiotic bacteria found in the intestines and oral mucosa of mammals (Vesth et al., 2013; Arif et al., 2008). While the rate of Veillonella species was over $1 \%$ in the control group created in the study, the rate of these bacterial species in the groups using Sinapic Acid and Ellagic Acid decreased below $0.1 \%$. It was observed that the Kineothrix alysoides species (Haas and Blanchard, 2017) in the Fimicutes phylum showed a significant increase in rats where Ellagic Acid and Sinapic Acid were used together. While the rate of Pseudoflavonifractor phocaeensis bacteria diagnosed by Ricaboni et al., (2017) was $0.02 \%$ in the bacteria in the control group, the rate of these bacteria was determined as $5.25 \%$ in the group where Ellagic Acid and Sinapic Acid were used together. Faecalibacterium prausnitzii (Bag et al., 2017), which lives as a commenseal bacterium in the intestinal system, increased in the group given Ellagic Acid, while it decreased in rats given Sinapic Acid compared to the control group. Romboutsia timonensis (Ricaboni et al., 2016) bacteria showed a decrease in rats other than the control group. Bacteria of the genus Blautia are microorganisms found in the intestines of mammals and have probiotic properties (Lin et al., 2021). It was determined that Blautia luti type bacteria increased especially in the group using Ellagic Acid. When stool samples of patients with the onset of rheumatoid arthritis were examined metagenomicly, it was observed that the number of Prevotella copri bacteria showed a large increase (Scher et al., 2013). In our study, when compared to the control group, Prevotella copri bacteria showed a significant increase in rats using Ellagic Acid, while this rate tended to decrease in rats using Sinapic Acid. In addition, it was determined that Rothia terrae species showed a significant decrease in rats treated with Ellagic Acid and Sinapic Acid.

While Actinomyces bovis (Mansouri et al., 2011), which rarely causes disease in humans, had a significant rate in the control group, it showed a great decrease in other rat groups. The genus Bacteroides is one of the dominant bacterial groups in the human gut and has an important role in maintaining a healthy intestinal ecosystem (Wexler AG, Goodman, 2017). There seems to be supporting data that commensal Parabacteroides distasonis found in the gut can be used as a probiotic in the treatment of metabolic functional disorders and in the regulation of host metabolism (Wang et al., 2019). In our study, it was determined that Bacteroides and Parabacteroides species increased in rat groups using Ellagic Acid. Intestinal microbiota; It affects immune development and homeostasis, host cell proliferation, neurological signaling, intestinal endocrine functions, bone density and energy mechanism (Lynch and Pedersen, 2016). The intestinal ecosystem is affected by many factors such as antibiotic use and diet. As a result of the deterioration of healthy intestinal flora, there is a decrease in colonization resistance and an increase in the rate of pathogens (Parlak and Dikmen, 2020). In recent years, the number of studies on intestinal metabolism has been increasing. It is especially important to investigate metabolic disorders that may occur as a result of inflammation of the intestinal microbiota. Because 
studies on the diagnosis and treatment of these health problems are an important point to increase the quality of life.

As a result of the metagenomic analyzes of the V3-V4 points of the 16S rDNA gene regions of the bacteria isolated from the intestine, it was observed that there were significant differences in the species diversity and microorganism ratio of the intestinal flora in the rat groups treated with Ellagic Acid and Sinapic Acid. As a result of this change, it was determined that there was an increase in the number of probiotic bacteria, especially in rats using Ellagic Acid, and a decrease in the number of some pathogens. On the other hand, the number of Lactobacillus species decreased in rats using Sinapic Acid compared to the control group, causing an abnormal increase in some Helicobacter species. It was observed that the number of some Lactobacillus species increased in the group in which Ellagic Acid and Sinapic Acid were given together.

\section{Conclusion}

One of the most important elements of living life is nutrition. While some living things carry out this work with their own adaptive mechanisms, many living things have to obtain food. Producer and consumer organisms need phenolic compounds such as Ellagic Acid and Sinapic Acid and their derivatives in order to perform some metabolic activities. In case of excess or deficiency of these compounds, troublesome situations may arise in terms of homeostasis. It can cause significant changes, especially in the species diversity of the microflora in our body. As a result of the study we have done, it has been determined that the Ellagic Acid and Sinapic Acid we use have a positive effect on the probiotic (Lactobacillus) microorganisms in the intestinal system. In addition, it has been determined that while it causes a decrease in the rate of some pathogenic microorganisms such as Streptococcus, it causes an increase in the number of Helicobacter. As a result, it shows that the phenolic compounds we use may have an effect that increases the mechanism of action of beneficial microorganisms and therefore facilitates digestion. It reveals the idea that further studies should be done on the increase in Helicobacter rate.

\section{Declarations}

Conflict of Interest The authors declare that there are no conficts of interest.

Ethical approval The experimental protocols of this study were approved by the Van Yüzüncü Yıl Animal Experiments Local Ethics Committee (Permit Number: 2019/03).

Informed consent Written informed consent was obtained from the author and all experiments were performed following approved guidelines and regulations.

\section{References}


Altındağ F, Ragbetli MÇ, Ozdek U, Koyun N, Alhalboosi JKI, Elasan S (2021) Combined treatment of sinapic acid and ellagic acid attenuates hyperglycemia in streptozotocin-induced diabetic rats. Food and Chemical Toxicology 156:112443. https://doi.org/10.1016/j.fct.2021.112443.

Arif N, Do T, Byun R, Sheehy E, Clark D, Gilbert SC, Beighton D (2008) Veillonella rogosae sp. nov., an anaerobic, Gram-negative coccus isolated from dental plaque. Int J Syst Evol Microbiol 58:581-584. https://doi.org/10.1099/ijs.0.65093-0.

Athukuri BL, Neerati P (2017) Enhanced Oral Bioavailability of Diltiazem by the Influence of Gallic Acid and Ellagic Acid in Male Wistar Rats: Involvement of CYP3A and P-gp Inhibition. Phytother Res 31:14411448. https://doi.org/10.1002/ptr.5873.

Ayres JS (2016) Cooperative Microbial Tolerance Behaviors in Host-Microbiota Mutualism. Cell 165:1323-1331. https://doi.org/10.1016/j.cell.2016.05.049.

Bag S, Ghosh TS, Das B (2017) "Faecalibacterium prausnitzii Isolated from the Gut of a Healthy Indian Adult". Genome Announcements. 5: e01286-17 https://doi.org/10.1128/genomeA.01286-17

Becattini S, Taur Y, Pamer EG (2016) Antibiotic induced changes in the intestinal microbiota and disease. Trends Mol Med; 22:458-478. https://doi.org/10.1016/j.molmed.2016.04.003.

Bin Jardan YA, Ansari MA, Raish M, Alkharfy KM, Ahad A, Al-Jenoobi Fl, et al., (2020). Sinapic Acid Ameliorates Oxidative Stress, Inflammation, and Apoptosis in Acute Doxorubicin-Induced Cardiotoxicity via the NF-kappaBMediated pathway. BioMed Res. Int.med Res Int 2020:3921796. https://doi.org/10.1155/2020/3921796.

Borre YE, Moloney RD, Clarke G, Dinan TG, Cryan JF (2014) The impact of microbiota on brain and behavior: mechanisms and therapeutic potential. Adv Exp Med Biol 817:373-403. https://doi.org/10.1007/978-1-4939-0897-4_17.

Ceci C, Lacal PM, Tentori L, De Martino MG, Miano R, Graziani G (2018) Experimental evidence of the antitumor, antimetastatic and antiangiogenic activity of ellagic acid. Nutrients 10:1756. https://doi.org/10.3390/nu10111756.

Chen C (2016), Sinapic Acid and Its Derivatives as Medicine in Oxidative Stress-Induced Diseases and Aging, Oxid Med Cell Longev, 2016:3571614. https://doi.org/10.1155/2016/3571614.

Chiu CY, Miller SA (2019) Clinical metagenomics. Nat Rev Genet 20:341-355.

https://doi.org/10.1038/s41576-019-0113-7

Deatherage BL, Cookson BT (2012) Membrane vesicle release in bacteria, eukaryotes, and archaea: a conserved yet underappreciated aspect of microbial life. Infect Immun 80:1948-1957. https://doi.org/10.1128/IAI.06014-11. 
Farbood Y, Rashno M, Ghaderi S, Esmaeil K, Sarkaki A, Rashidi K, Rashno M, Badavi M (2019) Ellagic acid protects against diabetes-associated behavioral deficits in rats: possible involved mechanisms. Life Sci 225:8-19. https://doi.org/10.1016/j.lfs.2019.03.078.

Fujimoto S, Amako K (1990) Guillain-Barré syndrome and Campylobacter jejuni infection Lancet 335:1350. https://doi.org/10.1016/0140-6736(90)91234-2.

Gill SR, Pop M, Deboy RT, Eckburg PB, Turnbaugh PJ, Samuel BS, Nelson KE (2006) Metagenomic analysis of the human distal gut microbiome. Science 312, 1355-1359.

https://doi.org/10.1126/science.1124234

Grundhofer P, Niemetz R, Schilling G, Gross GG (2001) Biosynthesis and subcellular distribution of hydrolyzable tannins. Phytochemistry 57:915-927. https://doi.org/10.1016/s0031-9422(01)00099-1.

Haas KN, Blanchard JL (2017) Kineothrix alysoides, gen. nov., sp. nov., a saccharolytic butyrate-producer within the family Lachnospiraceae. Int J Syst Evol Microbiol 67:402-410.

https://doi.org/10.1099/ijsem.0.001643.

Hameed H, Aydın S, Başaran N (2016) Sinapic Acid: Is It Safe for Humans? FABAD J Pharm Sci 41:3949.

Iglesiasa DE, Cremoninia E, Fraga CG, Oteiza PI (2020) Ellagic acid protects Caco-2 cell monolayers against inflammation-induced permeabilization. Free Radical Biology and Medicine 152:776-786. https://doi.org/10.1016/j.freeradbiomed.2020.01.022.

Lawson PA, Foster G, Falsen E, Ohlén M, Collins MD (1999) Vagococcus lutrae sp. nov., isolated from the common otter (Lutra lutra). Int J Syst Bacteriol 49:1251-1254 https://doi.org/10.1601/tx.5565.

Lefterova MI, Suarez CJ, Banaei N, Pinsky BA (2015) Next-generation sequencing for infectious disease diagnosis and management: a report of the association for molecular pathology. J Mol Diagn 17:623634. https://doi.org/10.1016/j.jmoldx.2015.07.004.

Levy M, Blacher E, Elinav E (2017) Microbiome, metabolites and host immunity. Curr Opin Microbiol 35:815. https://doi.org/10.1016/j.mib.2016.10.003.

Lin Z, Ye W, Zu X, Xie H, Li H, Li Y, Zhang W (2018). Integrative metabolic and microbial profiling on patients with spleen-yang-deficiency syndrome. Sci Rep 8(1):6619. https://doi.org/10.1038/s41598-01824130-7.

Liu X, Mao B, Gu J, Wu J, Cui S, Wang G, Zhao J, Zhang H, Chen W (2021) Blautia-a new functional genus with potential probiotic properties? Gut Microbes 13:1875796 https://doi.org/10.1080/19490976.2021.1875796. 
Lozupone C, Faust K, Raes J, Faith JJ, Frank DN, Zaneveld J et al., (2012) Identifying genomic and metabolic features that can underlie early successional and opportunistic lifestyles of human gut symbionts. Genome Res 22(10):1974-1984. https://doi.org/10.1101/gr.138198.112.

Lynch SV, Pedersen $O$ (2016) The human intestinal microbiome in health and disease. N Eng J Med 375:2369-2379. https://doi.org/10.1056/NEJMra1600266.

Makola D, Peura DA, Crowe SE (2007) Helicobacter pylori infection and related gastrointestinal diseases. Journal of Clinical Gastroenterology, 41:548-558. https://doi.org/10.1097/MCG.0b013e318030e3c3

Mansouri P, Farshi S, Khosravi A, Naraghi ZS (2011). "Primary cutaneous actinomycosis caused by Actinomyces bovis in a patient with common variable immunodeficiency". Journal of Dermatology. 38:911-915. https://doi.org/10.1111/j.1346-8138.2010.01165.x.

Morgan XC, Huttenhower C (2014) Meta'omic analytic techniques for studying the intestinal microbiome. Gastroenterology 146:1437-1448. https://doi.org/10.1053/j.gastro.2014.01.049.

Parlak L, Dikmen D (2020) Besin kaynaklı hastalıklarda intestinal mikrobiyotanın önemi. Türk Hijyen ve Deneysel Biyoloji Dergisi 77:497-508. https://doi.org/10.5505/TurkHijyen.2019.54926

Peng M, Biswas D (2017) Short chain and polyunsaturated fatty acids in host gut health and foodborne bacterial pathogen inhibition. Crit Rev in Food Sci and Nutr 57:3987-4002.

https://doi.org/10.1080/10408398.2016.1203286

Polce SA, Burke C, França LM, Kramer B, Paes AMA, Sepulveda MAC (2018) Ellagic Acid Alleviates Hepatic Oxidative Stress and Insulin Resistance in Diabetic Female Rats. Nutrients 10:531. https://doi.org/10.3390/nu10050531.

Raish M, Ahmad A, Ansari MA, Ahad A, Al-Jenoobi Fl, Al-Mohizea AM, Khan A, Ali N (2018) Sinapic acid ameliorates bleomycin-induced lungfibrosis in rats. Biomed Pharmacother 108:224-231. https://doi.org/10.1016/j.biopha.2018.09.032.

Raish M, Shahid M, Bin Jardan YA, Ansari MA, Alkharfy KM, Ahad A, Abdelrahman IA, Ahmad A and AlJenoobi FI (2021) Gastroprotective Effect of Sinapic Acid on Ethanollnduced Gastric Ulcers in Rats: Involvement of Nrf2/HO-1 and NF-Kb Signaling and Antiapoptotic Role. Front Pharmacol 12:622815. https://doi.org/10.3389/fphar.2021.622815.

Ricaboni D, Mailhe M, Benezech A, Andrieu C, Fournier PE, Raoult D (2017) 'Pseudoflavonifractor phocaeensis' gen. nov., sp. nov., isolated from human left colon. New Microbes New Infect 17:15-17. https://doi.org/10.1016/j.nmni.2016.12.012.

Ricaboni D, Mailhe M, Khelaifia S, Raoult D, Million M (2016) Romboutsia timonensis, a new species isolated from human gut New Microbes and New Infections 12:6-7. https://doi.org/10.1016/j.nmni.2016.04.001. 
Rios JL, Giner RM, Marin M, Recio MC (2018) A pharmacological update of ellagic acid. Planta Med 84:1068-1093. https://doi.org/10.1055/a-0633-9492.

Rooks MG, Garrett WS (2016) Gut microbiota, metabolites and host immunity. Nat Rev Immunol 16:341352. https://doi.org/10.1038/nri.2016.42.

Scher JU, Sczesnak A, Longman RS, Segata N, Ubeda C, Bielski C, et al., (2013) Expansion of intestinal Prevotella copri correlates with enhanced susceptibility to arthritis. Elife 2:e01202. https://doi.org/10.7554/eLife.01202.

Sekirov I, Russell SL, Antunes LCM, et al., (2010) Gut microbiota in health and disease. Physiol Rev 90:859-904. https://doi.org/10.1152/physrev.00045.2009.

Sorbara MT, Pamer EG (2019) Interbacterial mechanisms of colonization resistance and the strategies pathogens use to overcome them. Mucosal Immunology 12:1-9. https://doi.org/10.1038/s41385-0180053-0.

Tarnecki AM, Burgos FA, Ray CL, et al., (2017) Fish intestinal microbiome: diversity and symbiosis unraveled by metagenomics. J Appl Microbiol 123:2-17. https://doi.org/10.1111/jam.13415.

Udayangani RMC, Dananjaya SHS, Nikapitiya C, Heo GJ, Lee J, Zoysa MD (2017) Metagenomics analysis of gut microbiota and immune modulation in zebra fish (Danio rerio) fed chitosan silver nanocomposites. Fish \& Shellfish Immunology 66:173-184. https://doi.org/10.1016/j.fsi.2017.05.018

Vesth T, Ozen A, Andersen SC, Kaas RS, Lukjancenko O, Bohlin J, et al., (2013). Veillonella, firmicutes: microbes disguised as Gram negatives. Stand. Genom. Sci 9:431-448.

https://doi.org/10.4056/sigs.2981345.

Wang K, Liao M, Zhou N, Bao L, Ma K, Zheng Z, Wang Y, Liu C, Wang W, Wang J, et al., (2019) Parabacteroides distasonis Alleviates Obesity and Metabolic Dysfunctions via Production of Succinate and Secondary Bile Acids. Cell Rep., 26:222-235. https://doi.org/10.1016/j.celrep.2018.12.028.

Wexler AG, Goodman AL (2017) An insider's perspective: Bacteroides as a window into the microbiome.Nat Microbiol 2:17026. https://doi.org/10.1038/nmicrobiol.2017.26.

Yan Q, Li J, Yu Y, et al., (2016) Environmental filtering decreases with fish development for the assembly of gut microbiota. Environ Microbiol 18:4739-4754. https://doi.org/10.1111/1462-2920.13365.

Zhou B, Li Q, Wang K, Chen P, Jiang S (2019) Ellagic acid attenuates streptozocin induced diabetic nephropathy via the regulation of oxidative stress and inflammatory signaling. Foof and Chemical Toxicology 123:16-27. https://doi.org/10.1016/j.fct.2018.10.036.

Zinatizadeh MR, Abedini F, Jafarpour M, Masoumalinejad Z (2017) Identification of Mycoplasma Muris Isolated from Vaginal Samples of NIH Mice. Mod Med Lab J 1:100-106. 


\section{Figures}

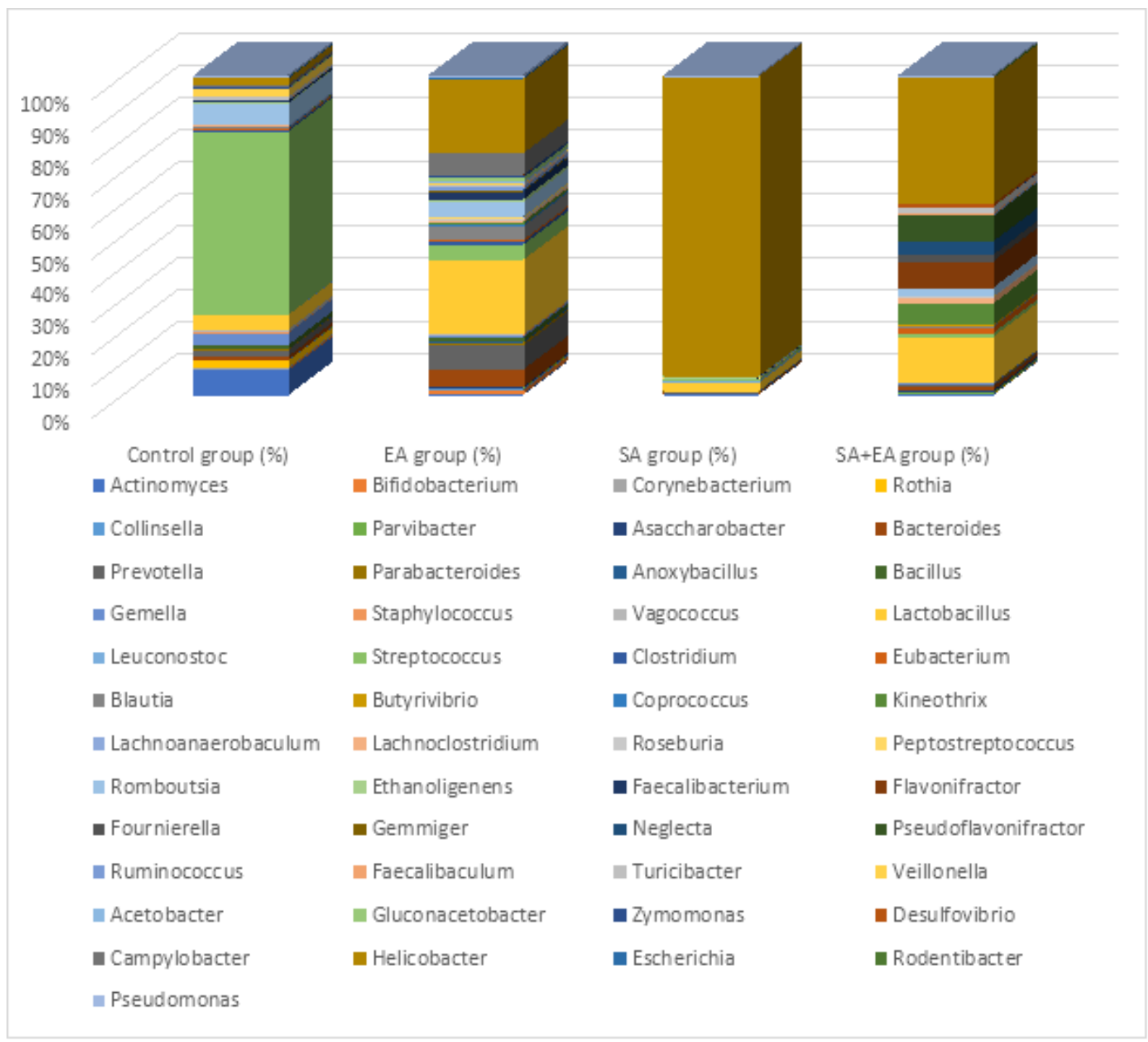

\section{Figure 1}

Taxonomic Column Chart for Species Level 